\title{
LITHIUM ABUNDANCES OF \\ SOLAR-TYPE STARS IN OPEN CLUSTERS
}

\author{
D.R. SODERBLOM
}

Space Telescope Science Institute

3700 San Martin Drive, Baltimore MD 21218 USA

\section{Introduction}

This paper discusses the lithium abundances of late- $F$ through mid- $K$ dwarfs in the nearby open clusters. That means that I am considering young disk stars, of course, with metallicities similar to the Sun's. I will not discuss the chasm seen among mid-F dwarfs. Li is not easily studied because only one spectroscopic feature is available and that feature is often weak. Thus high spectroscopic resolution and good signal to noise are needed. But $\mathrm{Li}$ is still the most easily studied of the light-element pentateuch $(\mathrm{D}, \mathrm{He}, \mathrm{Li}$, $\mathrm{Be}, \mathrm{B})$.

The few clusters now accessible to large telescopes at high spectroscopic resolution include the Hyades, Pleiades, $\alpha$ Persei, as well as smaller samples in a few others (such as Coma, M67, NGC 752, the Ursa Major Group). The samples within clusters are often substantial, and allow one to examine differences in $\mathrm{Li}$ between stars of the same mass, age, and composition. However the total number of clusters observed is still small, so one must assume that each cluster is fully representative for its age and composition.

Much recent work on $\mathrm{Li}$ in stars has been for Population II objects because of the importance of $\mathrm{Li}$ for cosmology. We study $\mathrm{Li}$ in Pop I stars for three reasons: First, to understand how the Galaxy has come to be enriched in Li since the Pop II stars formed. Second, to understand the stellar physics behind Li depletion in Pop I stars as a means to understanding what happens in the outer layers of such a star over time. Third, to apply that understanding of the stellar physics to Pop II stars so we can correctly infer the Big Bang Li abundance. 


\section{The Hyades}

The Hyades is not straightforwardly compared to the Sun because of its greater-than-solar metallicity, but in all other respects the Hyades is the best-studied case because its members are near, hence bright, and other studies provide essential ancillary information, such as membership and duplicity and rotation rates and activity levels for its solar-type stars.

The relationship between $\mathrm{Li}$ abundance $\left(N_{\mathrm{Li}}\right)$ and $T_{\text {eff }}$ for the Hyades is mostly a very tight one, with little scatter. There is a steady and sharp decline of $N_{\mathrm{Li}}$ with decreasing $T_{\text {eff }}$ (decreasing mass), consistent with the idea that cooler stars deplete Li faster because of their deeper convective envelopes. The stars that deviate most from the mean relation are nearly all binaries, leading to the suggestion that duplicity, especially the presence of a close companion, influences a star's ability to deplete its Li.

\section{The Pleiades}

In contrast to the tidiness seen in the Hyades, the Pleiades is chaotic. Especially interesting is the large spread in $N_{\mathrm{Li}}$ for stars with $T_{\text {eff }}$ and cooler. The origins of this spread are not clear, but it appears to be correlated with rotation (in the sense that the most rapidly rotating stars have the most $\mathrm{Li}$ ) and with activity (just because activity and rotation are correlated, if for no other reason). Star spots can play some role in creating an apparent spread in $N_{\mathrm{Li}}$ even when stars of the same mass have the same intrinsic abundance, but the coverage of spots needed to explain the most egregious cases can exceed 70 to 80

Recent Keck observations show the Li-rotation correlation to break down for mid- $K$ dwarfs.

\section{Other Indication About Li from Clusters}

All the clusters observed show some stars that are "drop-outs," i.e. stars with $N_{\mathrm{Li}}$ significantly lower that other stars of the same color in that cluster. For example, some stars in $\alpha$ Persei have $N_{\mathrm{Li}}$ even lower than stars of their color in the much older Hyades, yet they have other earmarks of genuine cluster membership. Can some agent lead to accelerated $\mathrm{Li}$ depletion in some stars, while that (or something else) retards Li depletion in other stars?

A comparison of clusters shows us that pre-main sequence Li depletion is important in accounting for what is seen but that some depletion must also occur on the main sequence, at least for solar-metallicity stars, and that the main sequence depletion mechanism slows down with age. 